\section{Moderated poster session 1}

\section{ASSESSMENT OF MARKERS OF CARDIAC TOXICITY FOLLOWING COMBINED TREATMENT OF CARDIOMYOCYTES WITH EPIRUBICIN AND TRASTUZUMA}

${ }^{1} \mathrm{C}$ Tonry, ${ }^{1} \mathrm{E}$ Mhic Donncha, ${ }^{1} \mathrm{H}$ Scott, ${ }^{2} \mathrm{M}$ Harbison, D Bell, ${ }^{1} \mathrm{C}$ Watson. ${ }^{1}$ Queens University Hospital Belfast, UK; ${ }^{2}$ Belfast Trust, UK

\subsection{6/heartjn|-2020-ICS.22}

Background Advancements in cancer therapy have significantly improved long-term survival rates of those with breast cancer. The addition of epirubicin treatment prior to standard trastuzumab treatment has been shown to slow progression of disease, reduce mortality and extend duration of survival in patients with HER2+ Breast Cancer. However, both drugs have off target cardiotoxic effects. Currently echocardiography is the only standardised diagnostic measure for detecting cardiac dysfunction. However, this is often after significant irreversible cardiac damage has occurred. There is an emerging need for blood-based biomarkers to aid in diagnosing subclinical cardiac dysfunction and to stratify those at risk prior to therapy.

Methods AC16 human cardiomyocytes were treated with Epirubicin $(2.6 \mathrm{ug} / \mathrm{ml})$ and Trastuzumab (150 ug/ml), both together and in monotherapy, over $10 \mathrm{hr}$ and $26 \mathrm{hr}$ timepoints. Cell viability was assessed via MTT cell viability assay. Protein and gene expression of Troponin I and BNP were assessed via western blot analysis and RT-PCR. Western blot analysis and fluorescent microscopy staining of oxidative stress markers was also carried out to elude to the potential mechanisms of cardiac damage.

Results Morphological changes occurred in all cells treated over 26 hrs, particularly with combined treatment. Cardiomyocytes treated with epirubicin alone showed the most significant reduction in cell viability compared to control ( $\mathrm{p} \leq$ $0.01 \%)$. Some increase in BNP and Troponin I expression was observed in cardiomyocytes treated with both epirubicin and trastuzumab. Pre-treatment of cardiomyocytes with recombinant BNP ameliorated chemotherapy-induced cell death in cardiomyocytes to some degree, however the effect was not significant.

Conclusion/Implications Combined treatment with epirubicin and trastuzumab exacerbates chemotherapy-induced cardiotoxicity. Troponin I and BNP are biomarkers that could be used as a diagnostic tool for prediction of subclinical chemotherapy-induced cardiotoxicty but further work is required to establish their true clinical utility.

\section{THE FIRST DESCRIPTION OF A SMARTPHONE-BASED EVALUATION OF THE CONJUNCTIVAL MICROCIRCULATION IN PATIENTS PRESENTING WITH ACUTE MYOCARDIAL INFARCTION}

${ }^{1} \mathrm{PF}$ Brennan, ${ }^{2} \mathrm{~A}$ Awuah, ${ }^{3} \mathrm{M}$ Jing, ${ }^{2} \mathrm{~A}$ McNeil, ${ }^{3} \mathrm{D}$ Finlay, ${ }^{2} \mathrm{~J}$ Mclaughlin, ${ }^{2} \mathrm{MA}$ Nesbit, ${ }^{4} \mathrm{E}$ Trucco, ${ }^{2} \mathrm{~T}$ Moore, ${ }^{1} \mathrm{MS}$ Spence. ${ }^{1}$ Royal Victoria Hospital, Belfast, UK; ${ }^{2}$ Ulster University, UK; ${ }^{3}$ NIBEC; ${ }^{4}$ VAMPIRE, University of Dundee, UK

10.1136/heartjnl-2020-ICS.23

Background Microcirculatory dysfunction and microvascular dysfunction occur early in the development of cardiovascular disease (CVD) with acute myocardial infarction (MI) being a late consequence of CVD. The conjunctival microcirculation is readily-accessible for quantitative assessment using a slit-lamp biomicroscope. We have previously reported the study of the conjunctival microcirculation in healthy volunteers and in patients with cyanotic congenital heart disease.

Methods We performed conjunctival microcirculatory assessment in a group of inpatients with acute type $1 \mathrm{MI}$ and in age/sex-matched healthy controls. Image acquisition and video capture was performed using an iPhone 6s combined with a slit-lamp biomicroscope. The conjunctival vessels in each hemisphere (temporal/nasal) of both eyes were studied. Microcirculatory parameters quantified included axial velocity, wall shear rate and blood volume flow.

Results Conjunctival microcirculatory assessment was assessed

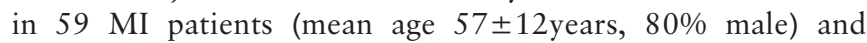

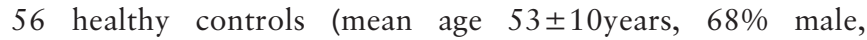
mean QRISK-3 score 8.1 $\pm 7.6 \%)$. STEMI and NSTEMI made up $36 \%(n=21)$ and $64 \%(n=38)$ of the MI patient group, respectively. Baseline characteristics are summarised in table 1.

A total of 4163 vessel segments (healthy control 1904 total, 34 per patient vs. MI 2259 total, 38 per patient) were analysed for the two groups. Mean conjunctival microvessel diameter was $21.41 \pm 7.57$ um for the controls which was significantly lower than the 22.32 \pm 7.66 um seen in MI patients $(p<0.0005)$. Axial velocity for the MI patients was significantly lower at $0.49 \pm 0.17 \mathrm{~mm} / \mathrm{s}$ compared to $0.53 \pm 0.15 \mathrm{~mm} / \mathrm{s}$ for the controls $(\mathrm{p}<0.0005$ (figure 1)). Wall shear rate was also significantly lower in the MI group (144.96 $\pm 88.45 \mathrm{~s}-1$ vs. $162 \pm 93 \mathrm{~s}-1, \mathrm{p}<0.0005$ (figure 2)). There was no significant difference in blood volume flow between the MI and controls $(154 \pm 124.8 \mathrm{pl} / \mathrm{s}$ vs. $152.6 \pm 124.4 \mathrm{pl} / \mathrm{s}, \mathrm{p} 0.84)$.

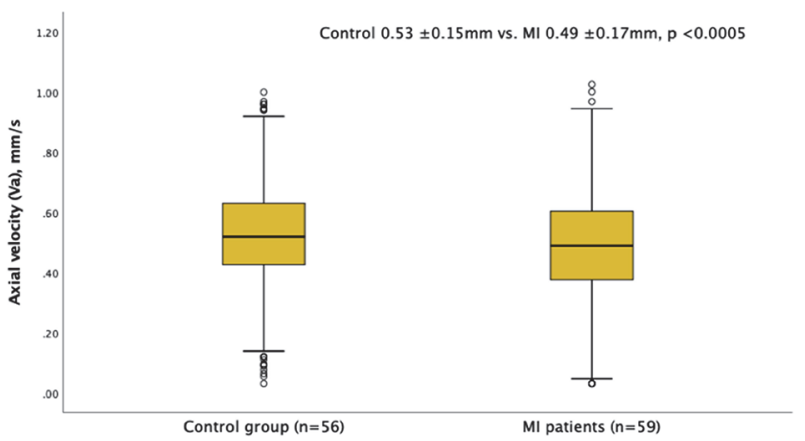

Abstract 23 Figure 1 Axial velocity

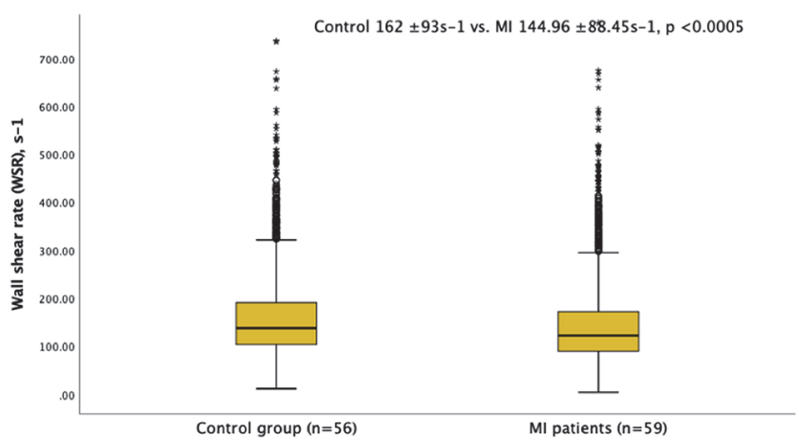

Abstract 23 Figure 2 Wall shear rate 